Artículo

\title{
Efecto de herbicidas preemergentes en el control de malezas y el desarrollo de cebolla bajo condiciones de fertirriego
}

\author{
Guadalupe Alfonso López-Urquídez \\ César Alfonso Murillo-Mendoza \\ Jesús Alonso Martínez-López \\ Felipe Ayala-Tafoya \\ Moisés Gilberto Yañez-Juárez \\ Carlos Alfonso López-Orona ${ }^{\S}$
}

Universidad Autónoma de Sinaloa-Facultad de Agronomía. Carretera Culiacán-El Dorado km 17.5, Culiacán, Sinaloa, México. CP. 80398. (alfonsolopezurquidez@uas.edu.mx; murillomendozacesaralfonso@ gmail.com; alonso_martinez1@hotmail.com; tafoya@uas.edu.mx; moisesyj@uas.edu.mx).

${ }^{\S}$ Autor para correspondencia: clopezorona@uas.edu.mx.

\section{Resumen}

La cebolla es de lento crecimiento, poca altura y raíces pequeñas. Por eso tiene desventaja ante las malezas; las cuales, si no son controladas afectan el rendimiento del cultivo. El objetivo del presente trabajo fue evaluar el efecto de la combinación de los herbicidas preemergentes Pendimethalin, Oxifluorfen, Oxadiazón, Acetoclor y Flumioxazin sobre la población de malezas y el rendimiento del cultivo. El experimento se estableció durante el ciclo otoño-invierno 2016-2017 en un predio en Culiacán, Sinaloa. Se tuvieron 16 tratamientos, incluido el testigo sin aplicación de herbicidas. El diseño experimental fue de bloques completos al azar, con tres repeticiones. En la variable densidad de plantas, el testigo sin aplicación fue el tratamiento con mayor densidad de malezas. Asimismo, la mejor combinación para inhibir la emergencia de malezas fue Acetoclor con Flumioxazin. Además, Flumioxazin solo o combinado se encuentra entre los tratamientos con menor densidad de malezas. En lo referente a la variable biomasa de malezas y eficiencia de los herbicidas, todos los tratamientos fueron superiores en eficacia al testigo. Además, la combinación de Pendimethalin con Acetoclor tuvo mejor comportamiento con $0.253 \mathrm{~kg} \mathrm{~m}^{-2}$. En la variable rendimiento del cultivo de cebolla e índice de maleza, las mejores combinaciones fueron Pendimethalin con Acetoclor y Oxifluorfen solo con 2.97 y $2.92 \mathrm{~kg} \mathrm{~m}^{-2}$, respectivamente. En contraparte el tratamiento Flumioxazin solo, tuvo menor rendimiento. Con respecto a la pérdida de efectividad biológica de herbicidas, se encontró que, en conjunto, por la pérdida de efectividad, aparecen en promedio 674 individuos de malezas ha ${ }^{-1}$ diariamente.

Palabras clave: cebolla, fertirriego, herbicida.

Recibido: mayo de 2020

Aceptado: junio de 2020 


\section{Introducción}

La cebolla es uno de los cultivos agrícolas más importantes a nivel mundial, ocupando el quinto lugar en volumen producción, con 117076053 t. Asimismo, en México, esta hortaliza ocupa el tercer lugar con una producción de 785862 t. La producción de esta hortaliza es limitada por la competencia ejercida por las malezas. Las malezas compiten con los cultivos de importancia agrícola por los recursos que se encuentran limitados, como lo son nutrientes, luz y espacio, principalmente (Froud-Wiliams, 2008), lo que puede provocar que disminuya el rendimiento de la cebolla entre 40 y $80 \%$ (Prakash et al., 2000).

Por eso es importante controlarlas, existiendo para ello varias alternativas, las cuales incluyen control cultural, el control manual, control químico y cubiertas con material orgánico (Olayinka y Etejere, 2015). Las malezas tienen mayor capacidad competitiva que las plantas cultivadas, como lo es velocidad de crecimiento, producción de semillas, etcétera, por lo que se requiere aplicar algún método de control (Jangre et al., 2018). Para lograr mayor eficiencia, el manejo del riego y de la fertilización debe ser integrado. La quimigación; a través, de un sistema de riego por goteo suministra las sustancias químicas directamente a la zona de interés. Debido a la precisión de aplicación, la quimigación puede ser menos peligrosa y usar menos productos químicos que otros métodos de aplicación. Muchos de los fertilizantes y pesticidas comunes pueden ser aplicados a través del sistema de riego por goteo (Shock y Welch, 2013; Gómez-Gálvez et al., 2019).

En el desarrollo de este proyecto se tomaron como base las siguientes premisas: a) en la aplicación del manejo integrado de malezas es importante que se entienda que la decisión que se tome para controlarlas puede afectar el rendimiento de la cebolla; b) la competencia en las primeras etapas del desarrollo de la cebolla puede causar pérdidas sustanciales de rendimiento del cultivo; c) que los herbicidas químicos son contaminantes, por lo cual se deben utilizar en las dosis más bajas posibles; d) que, al evaluar el efecto de las malezas en los cultivos, se debe tomar en cuenta las densidades de las poblaciones de las malezas, el tiempo de emergencia y el tiempo de remoción de las mismas; y e) que los herbicidas pueden causar fitotoxicidad a la cebolla, por lo cual es necesario determinar la dosis del producto, la formulación del mismo y cuál es la etapa fenológica del cultivo más apropiada para su aplicación. Algunos herbicidas contienen solo algún ingrediente activo, sin embargo, la mayoría son mezclas de al menos dos ingredientes activos.

Además, en la búsqueda de tener mayor eficacia en el control de malezas, se pueden realizar mezclas de dos o más herbicidas que han sido formulados independientemente uno del otro; sin embargo, algunas mezclas pueden ser antagónicas que causen daños a los cultivos o que disminuya la eficacia en el control de algunas malezas (Caseley, 1996). Con respecto al control químico se plantean diferentes alternativas, una de las más comunes es el uso de herbicidas.

El objetivo del trabajo de investigación fue determinar el efecto de herbicidas de acción preemergente en el control de malezas y el desarrollo del cultivo de cebolla bajo sistema de fertirriego. Las variables de respuesta a las diferentes combinaciones de herbicidas fueron: la emergencia de plantas de malezas a lo largo de un gradiente temporal, la producción de biomasa de malezas y el rendimiento del cultivo. Con esto se buscó una mejor solución para controlar las malezas en el cultivo de cebolla bajo sistema de riego por goteo. 


\section{Materiales y métodos}

\section{Ubicación geográfica del experimento}

El experimento se realizó durante el ciclo otoño-invierno 2016-2017 en un predio del Valle de San Lorenzo en Culiacán, Sinaloa, ubicado a los $24^{\circ} 27^{\prime} 27.53^{\prime}$ ' latitud norte, $107^{\circ} 16^{\prime}$ '02.32' ' longitud oeste del meridiano de Greenwich y una altitud de $38 \mathrm{~m}$ (Google Earth, 2018).

Características físicas donde se desarrolló el experimento. Se determinaron las características físicas del suelo mediante análisis de laboratorio, a partir de las cuales se estimó la lámina de riego, como se muestra en el Cuadro 1.

Cuadro 1. Características físicas del suelo para calcular la lámina de riego.

\begin{tabular}{cc}
\hline Características & Valor \\
\hline Arcilla (\%) & 27.96 \\
Limo (\%) & 23.44 \\
Arena (\%) & 48.06 \\
Capacidad de Campo (\%) & 21.2 \\
Punto de marchitez permanente $(\%)$ & 10.6 \\
Densidad aparente $\left(\mathrm{g} \mathrm{cm}^{-3}\right)$ & 1.282 \\
Distancia entre surcos $(\mathrm{cm})$ & 80 \\
\hline
\end{tabular}

\section{Determinación de las necesidades de agua de la cebolla}

Para estimar la distribución de los riegos a lo largo del ciclo del cultivo, se realizó el cálculo del uso consuntivo de la cebolla adecuado a las condiciones de la fecha de siembra y de las condiciones ambientales de la zona del experimento. Para ello se tomó como base la estación meteorológica más cercana al estudio, que en este caso fue la estación que se ubica en la Comunidad de Quilá, Culiacán, Sinaloa (México), como se observa en el Cuadro 2.

Cuadro 2. Uso consuntivo de la cebolla en Quilá, Sinaloa.

\begin{tabular}{cccccccc}
\hline Mes & $\begin{array}{c}\text { Precipitación } \\
(\mathrm{mm})\end{array}$ & $\begin{array}{c}\text { Temperatura } \\
\left({ }^{\circ} \mathrm{C}\right)\end{array}$ & $\mathrm{Kt}$ & $\mathrm{P}$ & $\mathrm{F}$ & $\mathrm{Kc}$ & $\mathrm{UC}(\mathrm{cm})$ \\
\hline Noviembre & 9.6 & 24.8 & 1.978 & 8.09 & 14.69 & 0.75 & 11.02 \\
Diciembre & 50.32 & 21.84 & 1.669 & 7.43 & 12.45 & 1.08 & 13.44 \\
Enero & 20.2 & 20 & 1.514 & 7.46 & 11.48 & 0.9 & 10.33 \\
Febrero & 21 & 20.2 & 1.59 & 7.58 & 11.4 & 0.7 & 7.98 \\
Total & & & & & & & 42.78 \\
\hline
\end{tabular}

$\mathrm{UC}=$ uso consuntivo; $\mathrm{Kt}=$ coeficiente de temperatura; $\mathrm{P}=$ porcentaje de horas-luz del mes; $\mathrm{F}=$ valor mensual que esta en función de la temperatura y el (\%) de h luz; Kc= coeficiente de desarrollo. 


\section{Fertilización de la cebolla}

Para la fertilización de la cebolla se utilizó el sistema de riego por goteo, por lo cual se aplicaron fertilizantes adecuados para este tipo de riego. La cantidad de fertilizantes se aplicó conforme se indica en el Cuadro 3.

Cuadro 3. Fertilizantes y dosis utilizada por hectárea en el cultivo de cebolla.

\begin{tabular}{|c|c|c|c|c|c|c|c|c|c|c|}
\hline Fuente & Formulación & $\begin{array}{l}\text { Fertilizante } \\
\quad(\mathrm{kg})\end{array}$ & $\begin{array}{c}\mathrm{N} \\
\text { total }\end{array}$ & Nitrato & Amonio & $\mathrm{P}$ & $\mathrm{K}$ & $\mathrm{Ca}$ & $\mathrm{Mg}$ & $\mathrm{S}$ \\
\hline Fosfato monopotásico & $0-52-34$ & 25 & & & & 13 & 8.51 & & & \\
\hline Nitrato de potasio & $12-00-46$ & 75 & 9 & 9 & & & 34.5 & & & 0.9 \\
\hline Nitrato de calcio & $15-00-00-30$ & 100 & 15 & 15 & & & & 30.1 & & \\
\hline Nitrato de magnesio & $11-00-00-16$ & 175 & 19.25 & 19.25 & & & & & 28 & \\
\hline Fosfonitrato de amonio & $33-04-00$ & 500 & 165 & 82.5 & 82.5 & 20 & & & & \\
\hline Ácido fosfórico & $0-52-0$ & 125 & & & & 65 & & & & \\
\hline Sulfato de potasio & $0-0-52-17$ & 125 & & & & & 65 & & & 21.3 \\
\hline Total & & & 208.3 & 125.8 & 82.5 & 98 & 108 & 30 & 28 & 22.2 \\
\hline
\end{tabular}

\section{Diseño experimental}

Se realizó un experimento cambiando por pares a cinco herbicidas recomendados en pre siembra para el control de malezas en cebolla. Se tuvieron 16 tratamientos, incluido el testigo sin aplicación de herbicidas, como se muestra en el Cuadro 4. El diseño experimental fue un experimento de bloques completos al azar, con tres repeticiones.

Cuadro 4. Tratamientos utilizados en el experimento.

\begin{tabular}{ccc}
\hline Tratamiento & Herbicida base & Herbicida complementario \\
\hline 1 & Pendimethalin & Oxifluorfen \\
2 & Pendimethalin & Acetochlor \\
3 & Pendimethalin & Oxadiazon \\
4 & Pendimethalin & Flumioxazin \\
5 & Pendimethalin & solo \\
6 & Oxifluorfen & Acetochlor \\
7 & Oxifluorfen & Oxadiazon \\
8 & Oxifluorfen & Flumioxazin \\
9 & Oxifluorfen & solo \\
10 & Acetochlor & Oxadiazon \\
11 & Acetochlor & Flumioxazin \\
12 & Acetochlor & solo \\
13 & Oxad & Flumioxazin \\
14 & Oxadiazon & solo \\
15 & Flumioxazin & solo \\
16 & Testigo sin aplicación & - \\
\hline
\end{tabular}


La unidad experimental estuvo constituida de cinco surcos de $3.5 \mathrm{~m}$ de longitud por $4 \mathrm{~m}$ de ancho. En cada cama se colocaron dos hileras de plantas con una separación $15 \mathrm{~cm}$ entre ellas y de $15 \mathrm{~cm}$ entre plantas. Cada surco presentó una cinta de riego por goteo, con separación entre goteros de 20 $\mathrm{cm}$ y un gasto de agua de $0.5 \mathrm{~L} \mathrm{~h}^{-1}$ por gotero.

Los datos sobre las malezas y del cultivo se obtuvieron de los tres surcos centrales dejando los surcos de las orillas como zona de amortiguamiento (efecto borde), así como un metro de cada extremo de la cama a evaluar. En el tratamiento testigo no se realizó control manual ni mecánico de las malezas. Las dosis aplicadas para cada tipo de herbicida fueron de acuerdo con la ficha técnica correspondiente. Las cuales se utilizaron tanto cuando el herbicida se aplicó solo o en combinación con otro (Cuadro 5).

Cuadro 5. Dosis de ingrediente activo aplicada por tipo de herbicida, según ficha técnica correspondiente.

\begin{tabular}{|c|c|c|c|}
\hline Ingrediente activo & Formulación & Concentración & $\begin{array}{l}\text { Dosis (ingrediente } \\
\text { activo) por ha }\end{array}$ \\
\hline Pendimethalin & $\begin{array}{c}\text { Prowl }^{\circledR} \mathrm{H}_{2} \mathrm{O} \text { (suspensión } \\
\text { capsulada) }\end{array}$ & $455 \mathrm{~g}_{\mathrm{ia} \mathrm{L}} \mathrm{L}^{-1}$ & $1365 \mathrm{~g}$ \\
\hline Oxifluorfen & $\begin{array}{c}\text { Goal Tender TM (suspensión } \\
\text { concentrada) }\end{array}$ & $480 \mathrm{~g}_{\text {ia L L }}-1$ & $360 \mathrm{~g}$ \\
\hline Acetoclor & $\begin{array}{c}\text { Harness }^{\circledR} \text { (concentrado } \\
\text { emulsionable) }\end{array}$ & $600 \mathrm{~g}_{\mathrm{ia} \mathrm{L}} \mathrm{L}^{-1}$ & $1500 \mathrm{~g}$ \\
\hline Oxadiazón & $\begin{array}{c}\text { Ronstar }^{\circledR} 25 \text { (concentrado } \\
\text { emulsionable) }\end{array}$ & $250 \mathrm{~g} \mathrm{ia} \mathrm{L}^{-1}$ & $250 \mathrm{~g}$ \\
\hline Flumioxazin & $\begin{array}{c}\text { SumiMax }{ }^{\circledR} \text { (gránulos } \\
\text { dispersables) }\end{array}$ & $510 \mathrm{~g}^{\mathrm{ia} \mathrm{kg}}{ }^{-1}$ & $153 \mathrm{~g}$ \\
\hline Testigo sin aplicación & & & - \\
\hline
\end{tabular}

En el Cuadro 6 se describe el modo de acción y familia química de los herbicidas utilizados en esta investigación.

Cuadro 6. Herbicidas de acuerdo con la familia química y modo de acción al que pertenecen.

\begin{tabular}{|c|c|c|}
\hline Ingrediente activo & Modo de acción & Familia química (grupo) \\
\hline Pendimethalin & Inhibición de la mitosis & Dinitroaniline \\
\hline Acetochlor & Inhibición de la mitosis & Chloroacetamide \\
\hline Oxifluorfen & $\begin{array}{l}\text { Inhibición de protoporfirinógeno } \\
\text { oxidasa (PPO) }\end{array}$ & Diphenylether \\
\hline Flumioxazin & $\begin{array}{l}\text { Inhibición de protoporfirinógeno } \\
\text { oxidasa (PPO) }\end{array}$ & N-phenylphthalimide \\
\hline Oxadiazón & $\begin{array}{l}\text { Inhibición de protoporfirinógeno } \\
\text { oxidasa (PPO) }\end{array}$ & Oxadiazole \\
\hline
\end{tabular}

WSSA (2018). 
El orden de establecimiento del cultivo fue el siguiente: 1) aplicación de la mezcla de herbicidas al suelo; 2) aplicación del riego. La cinta de riego con emisores dispuestos cada $15 \mathrm{~cm}$, con un gasto de $0.5 \mathrm{~L} \mathrm{~h}^{-1}$; 3) tratamiento del bulbillo con fungicida (tiabendazol); y 4) trasplante del bulbillo.

\section{Densidad de malezas}

Para estimar la densidad de malezas, se contó el número de malezas del total de especies por $\mathrm{m}^{2}$, se realizó el conteo de malezas cada semana, para ello se consideraron los $6 \mathrm{~m}^{2}$ centrales de cada unidad experimental (constituida de $14 \mathrm{~m}^{2}$ ), para lo cual, se formaron 6 cuadros de $1 \mathrm{~m}^{2}$ cada uno con hilo. Como criterio de inclusión se consideró que el tallo de la maleza estuviera dentro del cuadro formado en la unidad experimental (Kalhapure et al., 2013).

\section{Biomasa de la maleza}

Para determinar la biomasa de malezas, estas fueron secadas en estufa de secado a $80{ }^{\circ} \mathrm{C}$ hasta obtener un peso constante de acuerdo con la técnica sugerida por Nielsen (2003). La cosecha de malezas se realizó al final del ciclo del cultivo. El peso seco de la planta se obtuvo usando una balanza electrónica (precisión de $0.1 \mathrm{mg}$ ).

\section{Eficiencia del control de malezas}

Esta variable se calculó de acuerdo con el procedimiento propuesto por Mani et al. (1973); Ramalingam (2013) a partir de la siguiente fórmula: $\mathrm{EFM} \%=\frac{\mathrm{BMc}-\mathrm{BMt}}{\mathrm{BMc}} * 100$, donde: $\mathrm{EFM}=$ eficiencia del control de la maleza $(\%)$ BMc= biomasa de las malezas $\left(\mathrm{g} \mathrm{m}^{-2}\right)$ en la parcela de control; $\mathrm{BMt}=$ biomasa de malezas $\left(\mathrm{g} \mathrm{m}^{-2}\right)$ en la parcela tratada.

Rendimiento del cultivo de cebolla. Se cosecharon y pesaron los bulbos de cada parcela útil, a partir de lo cual se calculó el índice de maleza, bajo el procedimiento propuesto por Gill y Kumar (1969); Ramalingam (2013) a partir de la siguiente fórmula: $I M=\frac{X-Y}{X} * 100$, donde: $X=$ rendimiento $\left(\mathrm{kg} \mathrm{ha}^{-1}\right)$ de la parcela sin competencia de malezas; $\mathrm{Y}=$ rendimiento $\left(\mathrm{kg} \mathrm{ha}^{-1}\right)$ de la unidad experimental del tratamiento evaluado.

\section{Pérdida de efectividad biológica de los herbicidas}

Esta variable se calculó con base en la densidad de malezas, medida a lo largo del tiempo del desarrollo del experimento. Con los datos obtenidos se realizó un análisis de regresión lineal (Zar, 2010), donde la variable independiente fue el tiempo (en semanas) y la variable dependiente fue la densidad de plantas.

\section{Análisis de datos}

Para analizar los datos se realizó una prueba de normalidad y homocedasticidad, para aplicar un análisis de varianza de la densidad, biomasa y rendimiento de la cebolla; sin embargo, en los dos primeros casos los datos no tuvieron distribución normal, a pesar de que fueron transformados. Con respecto al rendimiento del cultivo, sí se tuvo una distribución normal, pero la prueba de homocedasticidad arrojó que las varianzas no eran iguales. Ante esta situación se optó por realizar un análisis estadístico no paramétrico, aplicando la prueba de Kruskal Wallis (Krebs, 1999). 
Para analizar la pérdida de efectividad biológica se realizó una regresión lineal, donde la variable independiente fue la combinación de los diferentes herbicidas preemergentes a lo largo de un gradiente temporal, mientras que la variable dependiente fue la densidad de plantas. En este análisis se utilizó el software estadístico Xlstat para Excel (Addinsoft, 2018).

\section{Resultados y discusión}

\section{Densidad de malezas}

El testigo sin aplicación fue el tratamiento con mayor densidad de malezas, con 213 plantas siendo estadísticamente diferente al resto de los tratamientos (Cuadro 7). Esto significa que los herbicidas aplicados tienen algún grado de control en la emergencia de las malezas. Asimismo, se muestra que la combinación más efectiva para inhibir la emergencia de malezas fue la combinación de Acetoclor con Flumioxazin. Además, llama la atención que Flumioxazin solo o combinado se encuentra entre los herbicidas con menor densidad de malezas.

Cuadro 7. Comparaciones múltiples por pares mediante el procedimiento de Dunn/prueba bilateral en el control de densidad de malezas.

\begin{tabular}{ccc}
\hline Tratamiento & Número de malezas promedio & Grupos \\
\hline Testigo sin aplicación & 213 & $\mathrm{~A}$ \\
Pendimethalin solo & 66 & $\mathrm{AB}$ \\
Oxifluorfen Oxadiazón & 58 & $\mathrm{AB}$ \\
Pendimethalin Oxadiazón & 50 & $\mathrm{AB}$ \\
Oxadiazón solo & 39 & $\mathrm{AB}$ \\
Pendimethalin Flumioxazin & 39 & $\mathrm{AB}$ \\
Pendimethalin Acetoclor & 37 & $\mathrm{AB}$ \\
Oxifluorfen solo & 35 & $\mathrm{AB}$ \\
Acetoclor solo & 34 & $\mathrm{AB}$ \\
Oxifluorfen Acetoclor & 33 & $\mathrm{AB}$ \\
Pendimethalin Oxifluorfen & 33 & $\mathrm{AB}$ \\
Flumioxazin solo & 30 & $\mathrm{AB}$ \\
Oxifluorfen Flumioxazin & 22 & $\mathrm{AB}$ \\
Acetoclor Oxadiazón & 19 & $\mathrm{AB}$ \\
Oxadiazón Flumioxazin & 18 & $\mathrm{AB}$ \\
Acetoclor Flumioxazin & 12 & $\mathrm{~B}$ \\
\hline
\end{tabular}

Lo anterior, sugiere que el herbicida más efectivo dentro de las combinaciones es Flumioxazin combinado con Acetoclor, sin diferir estadísticamente de todos los tratamientos químicos. Flumioxazin, combinado con Acetoclor mejora su acción debido a que pertenecen a grupos con distinto modo de acción actuando mejor sobre determinadas especies de malezas. 
Al respecto, Labrada y Parker (1996), señalan que en el control de malezas en cebolla se utilizan diferentes tipos de herbicidas, dependiendo de su tipo de control. Sin embargo, se deben considerar las otras variables evaluadas en esta investigación para concluir cuál es la mejor combinación de herbicidas.

\section{Biomasa de las malezas y eficiencia de los herbicidas}

El análisis arrojó que todos los tratamientos fueron mejor que el testigo. Además, se muestra que el tratamiento Pendimethalin más Acetoclor tuvo mejor comportamiento con respecto a la variable biomasa de las malezas con $0.253 \mathrm{~kg} \mathrm{~m}^{-2}$ (Cuadro 8).

Cuadro 8. Comparaciones múltiples por pares mediante el procedimiento de Dunn/prueba bilateral y eficiencia en el control de malezas.

\begin{tabular}{cccc}
\hline Tratamiento & $\begin{array}{c}\text { Medias LS }(\text { peso seco } \\
\text { malezas })\left(\mathrm{kg} \mathrm{m}^{-2}\right)\end{array}$ & Eficiencia & Grupos \\
\hline Testigo sin aplicación & 1.347 & 0 & $\mathrm{~A}$ \\
Pendimethalin solo & 1.284 & 4.693 & $\mathrm{AB}$ \\
Pendimethalin Oxifluorfen & 1.107 & 17.856 & $\mathrm{AB}$ \\
Oxadiazón solo & 1.092 & 18.969 & $\mathrm{AB}$ \\
Pendimethalin Oxadiazón 1.047 & 22.31 & & $\mathrm{AB}$ \\
Oxifluorfen Acetoclor & 1.011 & 24.948 & $\mathrm{AB}$ \\
Oxadiazón Flumioxazin & 0.967 & 28.247 & $\mathrm{AB}$ \\
Acetoclor Oxadiazón & 0.958 & 28.866 & $\mathrm{AB}$ \\
Pendimethalin Flumioxazin & 0.873 & 35.175 & $\mathrm{AB}$ \\
Flumioxazin solo & 0.778 & 42.268 & $\mathrm{AB}$ \\
Acetoclor solo & 0.731 & 45.773 & $\mathrm{AB}$ \\
Acetoclor Flumioxazin & 0.667 & 50.515 & $\mathrm{AB}$ \\
Oxifluorfen solo & 0.536 & 60.206 & $\mathrm{AB}$ \\
Oxifluorfen Oxadiazón & 0.478 & 64.536 & $\mathrm{AB}$ \\
Oxifluorfen Flumioxazin & 0.319 & 76.289 & $\mathrm{AB}$ \\
Pendimethalin Acetoclor & 0.253 & 81.196 & $\mathrm{~B}$ \\
\hline
\end{tabular}

Una de las razonas para que la combinación de Pendimethalin con Acetoclor tuviera menos peso de la maleza puede ser que presentan mecanismos de acción diferentes, puesto que el primero inhibe el ensamblaje de los micro túbulos los cuales son parte del esqueleto de las células, donde tienen múltiples funciones.

Mientras que Acetoclor pertenece al grupo que inhibe la división celular (Megías et al., 2019). Además, existe una combinación entre los dos tipos de herbicidas, puesto que Pendimetalin inhibe principalmente el desarrollo de la raíz, mientras que Acetoclor es absorbido principalmente por el coleoptilo y el hipocotilo. 
También se encontró que algunos herbicidas fueron más eficientes actuando solo que en combinación con otro, tal es el caso donde Oxifluorfen solo fue mejor que combinado con Acetoclor. Al respecto en un estudio realizado por Villalba et al. (2010) en el control de malezas en eucalipto, encontró que la combinación de Oxifluorfen con Acetoclor fue menos eficiente que Oxifluorfen solo, en el control de pasto Digitaria sanguinalis.

\section{Rendimiento del cultivo de cebolla e índice de maleza}

En el Cuadro 9 se muestra que las mejores combinaciones en el rendimiento de la cebolla fue Pendimethalin más Acetoclor, así como Oxifluorfen solo con 2.97 y $2.92 \mathrm{~kg} \mathrm{~m}^{-2}$ respectivamente. En contraparte el tratamiento con Flumioxazin solo fue el herbicida donde se presentó menor rendimiento.

Cuadro 9. Comparaciones múltiples por pares mediante el procedimiento de ConoverIman/Prueba bilateral en rendimiento de cebolla.

\begin{tabular}{cccc}
\hline Categoría & $\begin{array}{c}\text { Medias LS } \\
\text { (rendimiento cebolla kg m}{ }^{-2} \text { ) }\end{array}$ & $\begin{array}{c}\text { Índice } \\
\text { de maleza }\end{array}$ & Grupos \\
\hline Pendimethalin Acetoclor & 2.97 & 16.86 & $\mathrm{~A}$ \\
Oxifluorfen solo & 2.92 & 18.23 & $\mathrm{~A}$ \\
Pendimethalin Oxifluorfen & 2.83 & 20.73 & $\mathrm{AB}$ \\
Oxifluorfen Acetoclor & 2.74 & 23.34 & $\mathrm{AB}$ \\
Acetoclor Oxadiazón & 2.62 & 26.48 & $\mathrm{ABC}$ \\
Pendimethalin Oxadiazón & 2.12 & 40.65 & $\mathrm{ABCD}$ \\
Pendimethalin solo & 1.88 & 47.21 & $\mathrm{ABCD}$ \\
Oxifluorfen Flumioxazin & 1.67 & 53.17 & $\mathrm{ABCD}$ \\
Acetoclor Flumioxazin & 1.58 & 55.69 & $\mathrm{ABCD}$ \\
Pendimethalin Flumioxazin & 1.55 & 56.53 & $\mathrm{ABCD}$ \\
Oxadiazón Flumioxazin & 1.4 & 60.7 & $\mathrm{ABCD}$ \\
Acetoclor solo & 1.37 & 61.64 & $\mathrm{ABCD}$ \\
Oxifluorfen Oxadiazón & 1.37 & 61.72 & $\mathrm{ABCD}$ \\
Oxadiazón solo & 1.06 & 70.35 & $\mathrm{BCD}$ \\
Flumioxazin solo & 0.9 & 74.68 & $\mathrm{CD}$ \\
Testigo sin aplicación & 0.61 & 83.01 & $\mathrm{D}$ \\
\hline
\end{tabular}

Llama la atención que Flumioxazin solo fue el tratamiento con el menor peso del bulbo. En este aspecto se debe considerar qué dosis y qué formulación del herbicida, puede causar toxicidad al cultivo dependiendo de su etapa fenológica (Akey y Sousa, 1985; Karnataka, 2007; Ramalingam et al., 2013). En el caso de este herbicida, la ficha técnica indica que el producto se debe aplicar unos tres días después del trasplante.

Esto significa que el herbicida estaba activo al momento de colocar el bulbillo sobre el suelo tratado con este herbicida, y que al surgir la raíz esta haya sido dañada por el herbicida. Además, el Programa de Manejo Integrado de la Universidad de California (Smith et al., 2019), señalan que el herbicida Flumioxazin se recomienda solo para ajo, no para cebolla. 
Por otra parte, los resultados también muestran que no usar herbicidas, ni otro método de control, redujo los rendimientos $83 \%$. Esto refuerza el planteamiento de Prakash et al. (2000), con respecto a la pérdida de rendimiento en el cultivo de cebolla, causada por la competencia con malezas.

\section{Pérdida de efectividad biológica de herbicidas}

Al realizarse el análisis de varianza de la regresión, se observó significancia estadística con un valor de $p=0.004839$. Asimismo, el valor de $\mathrm{R}^{2}$ fue de 0.888 y un valor de la pendiente de la regresión de 0.6477 (Figura 1), lo que equivale a 674 nuevas plantas diarias por hectárea.

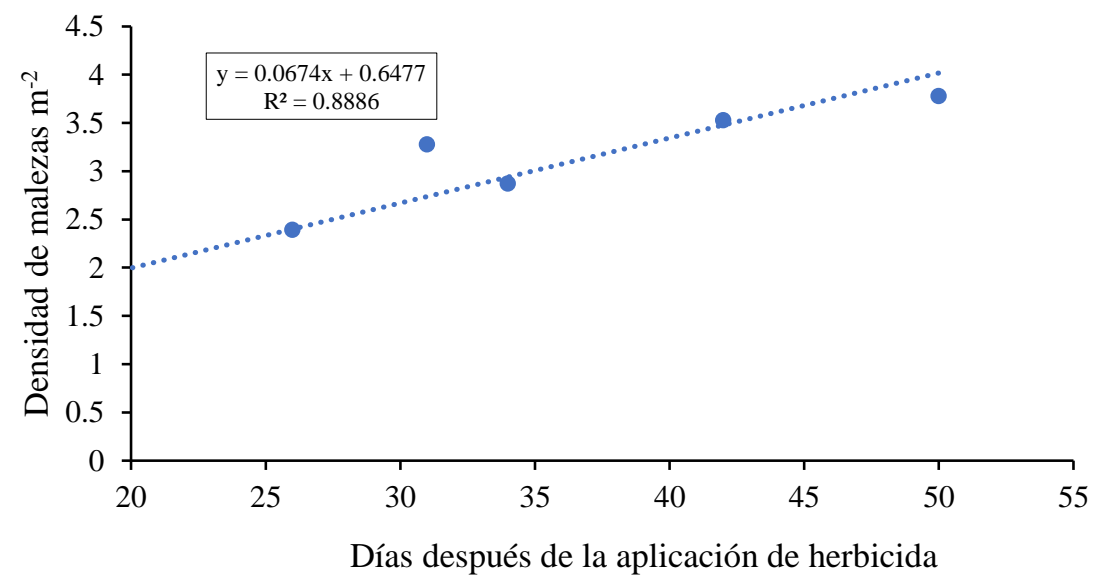

Figura 1. Relación entre el tiempo de aplicación de los herbicidas y el surgimiento de nuevas malezas.

En cuanto a la densidad de las malezas a través del tiempo se observó que en todos los tratamientos disminuye y luego hay un repunte de la densidad (Figura 2). Lo cual puede deberse a que las que emergen durante los primeros días después de la aplicación de los herbicidas, nacen débiles debido a la acción de estos últimos. Otra razón puede ser la competencia por espacio entre individuos de la misma especie y entre ellas (Begon et al., 2006).

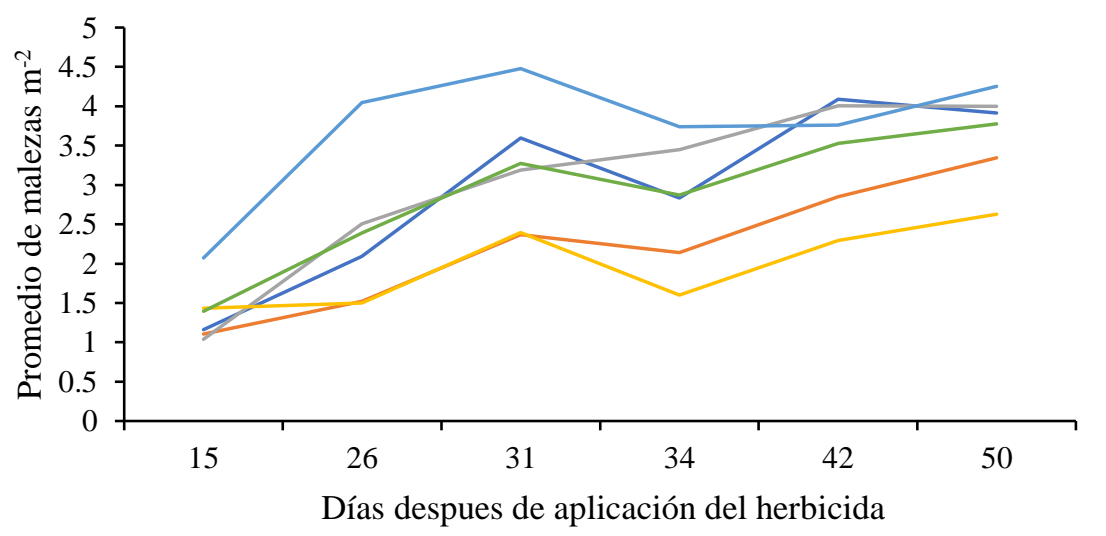

\begin{tabular}{|ll|}
\hline Oxifluorfen combinado & Acetoclor combinado \\
Oxadiazón combinado & Flumioxazin combinado \\
Pendimethalin combinado & Promedio general \\
\hline
\end{tabular}

Figura 2. Eficiciencia de los herbicidas días después de la aplicación de los herbicidas. 
El surgimiento de nuevos individuos de malezas, aparte de estar relacionado con la pérdida de residualidad, también está asociado a las características de las mismas, ya que tienen un proceso de germinación discontinuo (Baker, 1974; Altieri, 1999), pues la duración de la latencia de la semilla de maleza varía de acuerdo con el biotipo y las condiciones de almacenamiento de las semillas en el suelo (Ferrero, 2004).

Esta pérdida de efectividad biológica refuerza el planteamiento de que después de un herbicida aplicado en pretrasplante se aplique otro después del trasplante. Asimismo, se muestra la conveniencia de realizar un control manual de las malezas (Kalhapure et al., 2013). Aunado a lo anterior, las diferentes combinaciones con Flumioxazin fueron los tratamientos que menor densidad de malezas presentaron, por lo cual sería de esperarse que eso se viera reflejado en mayor peso de las cebollas al eliminarse parte de la competencia; sin embargo, no fue así.

Esto pudiera deberse a que este herbicida pudo haber causado fitotoxicidad en las plantas de cebolla. Al respecto, en la ficha técnica de este producto (Valent, 2017) se indica que Flumioxazin se debe aplicar sin combinarlo con otros herbicidas. También se debe tener en cuenta que la dosis y la formulación del herbicida, puede causar toxicidad al cultivo dependiendo de su etapa fenológica (Akey y Sousa, 1985; Karnataka, 2007; Ramalingam, et al., 2013).

\section{Conclusiones}

La combinación Acetoclor con Flumioxazin fue la que tuvo mejor en control en la densidad de las malezas, siendo este último herbicida el que presenta menor densidad de malezas. En la variable Biomasa de malezas y eficiencia de herbicidas, la combinación de Pendimethalín con Acetoclor presenta el mejor control, debido probablemente a que tienen diferente modo de acción en la emergencia de las malezas. Lo cual favoreció al rendimiento de la cebolla.

\section{Agradecimientos}

A la Universidad Autónoma de Sinaloa, por el financiamiento al trabajo de investigación a través del proyecto 'Efecto de herbicidas de acuerdo con su modo de acción en el desarrollo de la cebolla (Allium cepa L.) bajo condiciones de fertirriego’ con clave PROFAPI-2015/154.

\section{Literatura citada}

Addinsoft. 2018. XLSTAT Software. Versión 2018. 451393. Addinsoft 1995-2018.

Akey, W. C. y Souza, V. 1985. Response of onion (Allium cepa) to Oxifluorfen during early seedling development. Can. J. Plant Sci. 65(2):357-362. Doi: https://doi.org/10.4141/ cjps85-050.

Altieri, M. A. 1999. Agroecología bases científicas para una agricultura sustentable. Editorial Nordan Comunidad. Montevideo. 325 p.

Baker, H. F. 1974. The evolution of weeds. Ann. Rev. Eco. Syst. 5(1):1-24.

Begon, M.; Harper, J. y Townsend, C. 2006. Ecology. From Individuals to Ecosystems. $4^{\text {ta }}$ edition. Blackwell Publishing. Oxford.

Caseley, J. C. 1996. Capítulo 10. Herbicidas en: manejo de malezas para países en desarrollo. Editado por Labrada, R., J. C. Caseley y C. Parker. FAO. Roma. 
Ferrero, A. 2004. Arroz-maleza, características biológicas y control en capítulo 2. Malezas problemáticas y su manejo en áreas cultivables y no cultivadas en (manejo de malezas para países en desarrollo Addendum I. Editado por R. Labrada FAO. ROMA.

Froud-Wiliams. 2008. Weeds Competition. En: weed management handbook. Editado por Robert E. L. Naylor Blackwell Science y Brithish Crop Protection Counc. 16-38 pp.

Gill, G. S. y Kumar, K. V. 1969. Weed index-A new method of reporting weed control trial. Indian Journal of Agronomy. 16(2):96-98.

Google Earth. 2018. US Depto. of State Geographer. Image Lansat. (O2018. ORIONME. CGoogle.

Gómez-Gálvez, F. J.; Hidalgo-Moya, J. C.; Vega-Macías, V.; Hidalgo-Moya, J. J. y RodríguezJurado, D. 2019. Reduced introduction of Verticillium dahliae through irrigation systems and accumulation in soil by injection of peroxygen-based disinfectants. Plant Pathology. 68(1):116-126. Doi: 10.1111/ppa.12917.

Jangre, N.; Omesh, T.; Gupta, C. R. y Pandey, P. 2018. Review on pre and post emergence herbicides against weeds, yield attributes and yield of onion. Int. J. Curr. Microbiol. App. Sci. 7(4):1222-1230. Doi.org/10.20546/ijcmas.2018.704.136.

Kalhapure, A. H.; Shete, B. T. y Bodake, P. S. 2013. Integrated weed management in onion (Allium cepa). Indian Journal of Agronomy. 58(3):408-411.

Karnataka, J. 2007. Physiological studies on weed control efficiency in direct sown onion. Agric. Sci. 20(2):375-376.

Krebs, C. J. 1999. Ecological methodology. Benjamin Cummings. 620 p.

Labrada, R. y Parker, C. 1996. Capítulo 1. El control de malezas en el contexto del manejo integrado de plagas. En: manejo de malezas para países en desarrollo. Editado por Labrada, R., J. C. Caseley y C. Parker. FAO. Roma.

Mani, V. S.; Mala, M. L.; Gautam, K. C. y Bhagavandas. 1973. Weed killing chemicals in potato. Cultivation Indian Farming. 23(8):17-18.

Megías, M. P.; Molist.; y Pombal, M. A. 2019. Atlas de histología vegetal y animal. La célula. http://mmegias.webs.uvigo.es/5-celulas/1-introduccion.php.

Nielsen, S. 2003. Food analysis laboratory manual. Kluwer Academic/Plenum Publishers, New York, USA.

Olayinka, B. U. y Etejere, E. O. 2015. Growth analysis and yield of two varieties of groundnut (Arachis hypogaea L.) as influenced by different weed control methods. Ind. J. Plant Physiol. 20(2):130-136. D0i: 10.1007/s40502-015-0151-x.

Prakash, V.; Pandey, A. K.; Singh, R. D. y Mani, V. P. 2000. Integrated weed management in winter onion (Allium cepa L.) under Mid-Hills conditions of North-Western Himalayas. Indian Journal of Agronomy. 45(4):816-821.

Ramalingam, S. P.; Chinnagounder, C.; Perumal, M. y Palanisamy, M. A. 2013. Evaluation of new formulation of oxifluorfen $(23.5 \% \mathrm{EC})$ for weed control efficacy and bulb yield in onion. American Journal of Plant Sciences. 4(4):890-895. Doi:/10.4236/ajps.2013.44109.

Sangeeta, K.; Shivnath, D.; Kumar, R. y Kavita. 2018. Herbicide mixture for enhancing weed control efficiency and yield of onion (Onion cepa L.) Int. J. Curr. Microbiol. App. Sci. 7(1):2710-2714.

SIAP. 2018. Anuario de estadístico de cultivos. http://www.siap.gob.mx/siembras-ycosechas $5 / 06 / 2018$.

Shock, C. C. y Welch, T. 2013. El riego por goteo: una introducción. En: técnicas para la agricultura sostenible. Editado por: Oregon State University. Corvallis, OR.

Smith, R. F.; Fennimore, S. A. y Orioff, S. 2019. UC-IPM pest management guidelines. Onion and Garlic. University of California IPM. http://ipm.ucanr.edu/PDF/PMG/pmgoniongarlic.pdf. 
Valent. 2017. Ficha técnica. SumiMax. Valent.

Villalba, J.; Montouto, C.; Cazaban, J.; Caraballo, P. y Betancur, O. 2010. Efecto del laboreo sobre la eficacia de herbicidas y el crecimiento de Eucalyptus spp. Agrociencia Uruguay. 14(2):45-54.

WSSA (Weed Science Society of America). 2018. Herbicide site of action (SOA). Classification List. http://wssa.net/wssa/weed/herbicides/.

Zar, J. A. 2010. Bioestatistical Analysis. $5^{\text {ta }}$ edition. Prentice-Hall, Englewood Cliffs. 